Mathematical Modelling And AnAlysis

Volume 18 Number 1, February 2013, 53-65

http://dx.doi.org/10.3846/13926292.2013.756833

(c) Vilnius Gediminas Technical University, 2013
Publisher: Taylor\&Francis and VGTU

http://www.tandfonline.com/TMMA

Print ISSN: 1392-6292

Online ISSN: 1648-3510

\title{
On a Second-Order Differential Problem with Fractional Derivatives of Order Greater than One
}

\section{Nasser-eddine Tatar}

\author{
King Fahd University of Petroleum and Minerals \\ Dhahran, Saudi Arabia \\ E-mail: tatarn@kfupm.edu.sa
}

Received December 14, 2011; revised November 6, 2012; published online February 1, 2013

\begin{abstract}
A second-order abstract problem with derivatives of non-integer order is investigated. The nonlinearity involves fractional derivatives between 1 and 2 . Existence and uniqueness of mild and classical solutions are established in appropriate spaces. This work extends similar works with or without a derivative of first order and also a work of the present author, where the order of the derivatives were between 0 and 1.
\end{abstract}

Keywords: Cauchy problem, cosine family, fractional derivative, mild solutions, secondorder abstract problem.

AMS Subject Classification: 26A33; 34K40; 35L90; 35L70; 35L15; 35L07.

\section{Introduction}

Of concern in this paper is the following problem

$$
\left\{\begin{array}{l}
u^{\prime \prime}(t)=A u(t)+f\left(t, u(t), t^{\gamma} D^{\beta} u(t)\right), \quad t>0 \\
u(0)=u^{0} \in X, \quad u^{\prime}(0)=u^{1} \in X
\end{array}\right.
$$

with $1<\beta<2$ and $\gamma \geq 0$. Here the prime denotes time differentiation and $D^{\beta}$ denotes fractional time differentiation (in the sense of Riemann-Liouville). The operator $A$ is the infinitesimal generator of a strongly continuous cosine family $C(t), t \geq 0$ of bounded linear operators in the Banach space $X$ and $f$ is a nonlinear function from $\mathbf{R}^{+} \times X \times X$ to $X, u^{0}$ and $u^{1}$ are given initial data in $X$.

This problem has been studied in case $\beta=0$ or 1 (and $\gamma=0$ ) (see for instance $[14,25]$ ). Well-posedness has been proved using different methods such as fixed point theorems and the theory of strongly continuous cosine families in Banach spaces. We refer the reader to $[25,26]$ for an introduction to the theory of the cosine family. Several results on classical solutions and mild 
solutions have been proved under different conditions on the nonlinearities and the initial data $[4,6,7,8,9,13,14,27]$. In case $\beta=1$, the natural underlying space where to look for mild solutions is the space of continuously differentiable functions.

Here we consider the case, where $1<\beta<2$. This work clearly extends the cases $\beta=0$ and $\beta=1$ and also the case $0<\beta<1$ studied by the present author in [24]. In that work we were not able to consider derivatives of higher order $(1<\beta<2)$. The reason is simple: the method adopted there used a differentiation by the chain rule of the nonlinearity $f$ which of course leads to a fractional derivative of order greater than 2 (namely of order $\beta+2$ ). This causes a problem of regularity and prevents the existence and continuity of the second derivative of $u$ at zero. In this work we prove solvability of the problem provided that the nonlinearity is an $E$-valued function (see (2.1) below) in a space of minimum regularity.

We mention here that fractional derivatives appear in many engineering problems. They arise naturally in many fields such as in probability, physics, astrophysics, chemical physics, anomalous diffusion, seismic analysis, finance, optic and signal processing, robust control, electromagnetism, biology, viscoelasticity, acoustics (see $[12,15,21,22,23]$ to cite but a few). In mechanics, for instance, fractional derivatives are more adequate to model the damping than the usual frictional or structural dampings (described by the time derivative of order one or the time derivative of the Laplacian, respectively). This has been clearly shown by experiments (see $[1,2,3])$. We can also cite the references $[5,10,17,18]$, where fractional derivatives appear in a natural way. In many situations the fractional derivatives appear in a simple and linear form so that some of the well-known methods (such as the Laplace transform) provide the explicit solutions. Here in this paper we consider a somewhat general nonlinear situation.

The next section of this paper contains some notation and preliminary results needed in our proofs. Section 3 treats the existence and uniqueness of a mild solution in an appropriate space. Section 4 is devoted to the existence and uniqueness of a classical solution.

\section{Preliminaries}

In this section we present some notation, assumptions and results needed in our proofs later.

Definition 1. The integral

$$
\left(I_{a+}^{\alpha} h\right)(x)=\frac{1}{\Gamma(\alpha)} \int_{a}^{x} \frac{h(t) d t}{(x-t)^{1-\alpha}}, \quad x>a
$$

is called the Riemann-Liouville fractional integral of $h$ of order $\alpha>0$ when the right side exists. Here $\Gamma$ is the usual Gamma function

$$
\Gamma(z):=\int_{0}^{\infty} e^{-s} s^{z-1} d s, \quad z>0
$$

and it is extended to negative values as well. 
Definition 2. The (left hand) Riemann-Liouville fractional derivative of $h$ of order $\alpha>0$ is defined by

$$
\left(D_{a}^{\alpha} h\right)(x)=\frac{1}{\Gamma(n-\alpha)}\left(\frac{d}{d x}\right)^{n} \int_{a}^{x} \frac{h(t) d t}{(x-t)^{\alpha-n+1}}, \quad x>a, n=[\alpha]+1
$$

whenever the right side is pointwise defined.

In particular

$$
\begin{aligned}
& \left(D_{a}^{\beta} h\right)(x)=\frac{1}{\Gamma(1-\beta)} \frac{d}{d x} \int_{a}^{x} \frac{h(t) d t}{(x-t)^{\beta}}, \quad x>a, 0<\beta<1 \\
& \left(D_{a}^{\beta} h\right)(x)=\frac{1}{\Gamma(2-\beta)}\left(\frac{d}{d x}\right)^{2} \int_{a}^{x} \frac{h(t) d t}{(x-t)^{\beta-1}}, \quad x>a, 1<\beta<2 .
\end{aligned}
$$

We will also need the following lemma which can be found in [15, 23].

Lemma 1. If $h(x) \in A C^{n}[a, b]:=\left\{\phi:[a, b] \rightarrow \mathbf{R}\right.$ and $\left.\left(D^{n-1} \phi\right)(x) \in A C[a, b]\right\}$, $\alpha>0$ and $n=[\alpha]+1$, then

$$
\begin{aligned}
\left(D_{a}^{\alpha} h\right)(x) & =\sum_{k=0}^{n-1} \frac{h^{(k)}(a)}{\Gamma(1+k-\alpha)}(x-a)^{k-\alpha}+\frac{1}{\Gamma(n-\alpha)} \int_{a}^{x} \frac{h^{(n)}(t) d t}{(x-t)^{\alpha-n+1}} \\
& :=\sum_{k=0}^{n-1} \frac{h^{(k)}(a)}{\Gamma(1+k-\alpha)}(x-a)^{k-\alpha}+\left({ }^{C} D_{a}^{\alpha} h\right)(x), \quad x>a .
\end{aligned}
$$

The expression $\left({ }^{C} D_{a}^{\alpha} h\right)(x)$ is known as the fractional derivative of order $\alpha$ in the sense of Caputo. Therefore this lemma provides a relationship between these two types of fractional derivatives. See $[11,15,19,20,22,23]$ for more on fractional derivatives and fractional integrals.

A one parameter family of bounded operators $C(t): X \rightarrow X, t \in \mathbf{R}$, where $X$ is a Banach space is called a strongly continuous cosine family if

(i) $C(s+t)+C(s-t)=2 C(s) C(t), s, t \in \mathbf{R}$,

(ii) $C(0)=I$,

(iii) $C(t) x$ is continuous in $t \in \mathbf{R}$ for fixed $x \in X$.

The infinitesimal generator of a strongly continuous family $C(t), t \in \mathbf{R}$ is the operator $A$ on $X$ defined by

$$
\begin{gathered}
A x:=\frac{d^{2}}{d t^{2}} C(0) x \\
D(A):=\{x \in X, C(t) x \text { is twice continuously differentiable }\} .
\end{gathered}
$$

The operator $A$ is assumed to satisfy

(H1) $A$ is the infinitesimal generator of a strongly continuous cosine family $C(t), t \in \mathbf{R}$, of bounded linear operators in the Banach space $X$. 
The associated sine family $S(t), t \in \mathbf{R}$ is defined by

$$
S(t) x:=\int_{0}^{t} C(s) x d s, \quad t \in \mathbf{R}, x \in X .
$$

It is known (see $[25,26])$ that there exist constants $M \geq 1$ and $\omega \geq 0$ such that

$$
|C(t)| \leq M e^{\omega|t|}, \quad t \in \mathbf{R} \quad \text { and } \quad\left|S(t)-S\left(t_{0}\right)\right| \leq M\left|\int_{t_{0}}^{t} e^{\omega|s|} d s\right|, \quad t, t_{0} \in \mathbf{R} .
$$

For simplicity we will write $|C(t)| \leq \tilde{M}$ and $|S(t)| \leq \tilde{N}$ on $I=[0, T]$ (of course $\tilde{M} \geq 1$ and $\tilde{N} \geq 1$ depend on $T)$.

If we define

$$
E:=\{x \in X: C(t) x \text { is once continuously differentiable on } \mathbf{R}\},
$$

then we have

Lemma 2 [see [25, 26]]. Assume that (H1) is satisfied. Then

(i) $S(t) X \subset E, t \in \mathbf{R}$,

(ii) $S(t) E \subset D(A), t \in \mathbf{R}$,

(iii) $\frac{d}{d t} C(t) x=A S(t) x, x \in E, t \in \mathbf{R}$,

(iv) if $x \in D(A)$, then $C(t) x \in D(A)$ and $\frac{d^{2}}{d t^{2}} C(t) x=A C(t) x=C(t) A x$, $t \in \mathbf{R}$.

Definition 3. A function $u \in C^{2}(I, X)$, such that $t^{\gamma} D^{\beta} u(t)$ exists and is continuous on $I$, is called a classical solution of (1.1) if $u(.) \in D(A)$, satisfies the equation in (1.1) and the initial conditions are verified.

Lemma 3 [see [25]]. Suppose that (H1) holds, $v: \mathbf{R} \rightarrow X$ a continuous function and $q(t)=\int_{0}^{t} S(t-s) v(s) d s$. The initial value problem $u^{\prime \prime}(t)=A u(t)+$ $v(t), t \in \mathbf{R}, u(0)=u^{0}, u^{\prime}(0)=u^{1}$ has a classical solution for every $u^{0} \in D(A)$ and $u^{1} \in E$ if and only if $q(t)$ is twice continuously differentiable for $t \in \mathbf{R}$. In particular, if $q(t)$ is twice continuously differentiable then $q(t) \in D(A), t \in \mathbf{R}$ and $A q(t)=q^{\prime \prime}(t)-v(t)$.

We mention here that, even for twice continuously differentiable functions $u$ the problem of singularity at zero of $D^{\beta} u$ remains an issue.

Definition 4. A continuous function $u$ such that $t^{\gamma} D^{\beta} u(t) \in C(I, X)$ and which satisfies the integro-differential equation

$$
u(t)=C(t) u^{0}+S(t) u^{1}+\int_{0}^{t} S(t-s) f\left(s, u(s), s^{\gamma} D^{\beta} u(s)\right) d s
$$

is called a mild solution of problem (1.1).

It is known from [27] that, in case of continuity of the nonlinearity, solutions of (1.1) are solutions of the more general problem (2.2). That is, they are also mild solutions. 


\section{Existence of Mild Solutions}

In this section we prove existence and uniqueness of a mild solution in the space

$$
C_{\beta, \gamma}^{R L}([0, T]):=\left\{v \in C([0, T]): t^{\gamma} D^{\beta} v \in C([0, T])\right\}, \quad \gamma \geq 0, \beta>0
$$

equipped with the norm $\|v\|_{\beta, \gamma}:=\|v\|_{C}+\left\|t^{\gamma} D^{\beta} v\right\|_{C}$, where $\|\cdot\|_{C}$ is the sup norm in $C([0, T])$. For the initial data we define

$$
E_{\beta, \gamma}:=\left\{x \in X: t^{\gamma} D^{\beta} C(t) x \text { is continuous on } \mathbf{R}^{+}\right\} .
$$

The assumption on $f$ is

(H2) $f: \mathbf{R}^{+} \times X \times X \rightarrow X$ is continuous and satisfies the Lipschitz condition

$$
\left\|f\left(t, x_{1}, y_{1}\right)-f\left(t, x_{2}, y_{2}\right)\right\| \leq F(t)\left(\left\|x_{1}-x_{2}\right\|+\left\|y_{1}-y_{2}\right\|\right)
$$

for $x_{1}, y_{1}, x_{2}, y_{2} \in X$ and some continuous real-valued function $F(t)$.

The next lemma can be found in [24] in case $0<\nu<1$.

Lemma 4. If $R(t)$ is a linear operator such that $I^{1-\nu} R(t) x \in C^{1}([0, T]), T>$ 0 , then, for $0<\nu<1$, we have

$$
D^{\nu} \int_{0}^{t} R(t-s) x d s=\int_{0}^{t} D^{\nu} R(t-s) x d s+\lim _{t \rightarrow 0^{+}} I^{1-\nu} R(t) x, \quad x \in X, t \in[0, T] .
$$

Proof. By Definition 2 and Fubini's theorem we have

$$
\begin{aligned}
D^{\nu} \int_{0}^{t} R(t-s) x d s= & \frac{1}{\Gamma(1-\nu)} \frac{d}{d t} \int_{0}^{t} \frac{d \tau}{(t-\tau)^{\nu}} \int_{0}^{\tau} R(\tau-s) x d s \\
= & \frac{1}{\Gamma(1-\nu)} \frac{d}{d t} \int_{0}^{t} d s \int_{s}^{t} \frac{R(\tau-s) x}{(t-\tau)^{\nu}} d \tau \\
= & \frac{1}{\Gamma(1-\nu)} \int_{0}^{t} d s \frac{\partial}{\partial t} \int_{s}^{t} \frac{R(\tau-s) x}{(t-\tau)^{\nu}} d \tau \\
& +\frac{1}{\Gamma(1-\nu)} \lim _{s \rightarrow t^{-}} \int_{s}^{t} \frac{R(\tau-s) x}{(t-\tau)^{\nu}} d \tau .
\end{aligned}
$$

These steps are justified by the assumption $I^{1-\nu} R(t) x \in C^{1}([0, T])$. Moreover, a change of variable $\sigma=\tau-s$ leads to

$$
\begin{aligned}
D^{\nu} \int_{0}^{t} R(t-s) x d s= & \frac{1}{\Gamma(1-\nu)} \int_{0}^{t} d s \frac{\partial}{\partial t} \int_{0}^{t-s} \frac{R(\sigma) x}{(t-s-\sigma)^{\nu}} d \sigma \\
& +\frac{1}{\Gamma(1-\nu)} \lim _{t \rightarrow 0^{+}} \int_{0}^{t} \frac{R(\sigma) x}{(t-\sigma)^{\nu}} d \sigma .
\end{aligned}
$$

This is the formula stated in the lemma.

Below we state and prove a similar result in case $1<\nu<2$. 
Lemma 5. Let $S(t)$ be the sine family associated with the cosine family $C(t)$ and $g$ be a continuous $E_{\nu-1}$-valued function $\left(E_{\nu-1}:=E_{\nu-1,0}\right.$, see (3.2)). Then, for $1<\nu<2$, we have

$$
D^{\nu} \int_{0}^{t} S(t-s) g(s) d s=\int_{0}^{t} D^{\nu-1} C(t-s) g(s) d s, \quad t \in[0, T] .
$$

Proof. By formula (2.122) in [22]

$$
D^{\rho}\left(\frac{d^{n} h(t)}{d t^{n}}\right)=D^{\rho+n} h(t)-\sum_{j=0}^{n-1} \frac{h^{(j)}(0) t^{j-\rho-n}}{\Gamma(1+j-\rho-n)}, \quad \rho>0,
$$

for $n=1$ we see that

$$
D^{\rho} h^{\prime}(t)=D^{\rho+1} h(t)-\frac{h(0) t^{-\rho-1}}{\Gamma(-\rho)}, \quad \rho>0 .
$$

Therefore, by the continuity of $S$ and $g$, the fact that $S(0)=0$ and Lemma 2

$$
\begin{gathered}
D^{\nu} \int_{0}^{t} S(t-s) g(s) d s=D^{\nu-1+1} \int_{0}^{t} S(t-s) g(s) d s=D^{\nu-1} \frac{d}{d t} \int_{0}^{t} S(t-s) g(s) d s \\
=D^{\nu-1} \int_{0}^{t} C(t-s) g(s) d s=\int_{0}^{t} D^{\nu-1} C(t-s) g(s) d s,
\end{gathered}
$$

where we have used the assumption that $g(t)$ is an $E_{\nu-1}$-valued function that is $I^{2-\nu} C(t) g(t) \in C^{1}([0, T])$ which allowed us to apply the previous lemma (Lemma 4 ) in the last step of (3.3). This completes the proof.

We are now ready to state and prove our first main result.

Theorem 1. Assume that (H1)-(H2) hold, $1<\beta<2$ and $\gamma \geq 0$. If $\left(u^{0}, u^{1}\right) \in$ $E_{\beta, \gamma} \times E_{\beta-1, \gamma}$ and $f$ is an $E_{\beta-1}$-valued continuous function, then there exist $T>0$ and a unique mild solution $u \in C_{\beta, \gamma}^{R L}([0, T])$ of problem (1.1).

Proof. First note that for $u \in C_{\beta, \gamma}^{R L}([0, T])$, the function

$$
\psi(t):=C(t) u^{0}+S(t) u^{1}+\int_{0}^{t} S(t-s) f\left(s, u(s), s^{\gamma} D^{\beta} u(s)\right) d s
$$

is continuous. Recalling that $S(0)=0$ and by Lemma 5 we infer that

$$
\begin{aligned}
D^{\beta} \psi(t) & =D^{\beta} C(t) u^{0}+D^{\beta} S(t) u^{1}+D^{\beta} \int_{0}^{t} S(t-s) f\left(s, u(s), s^{\gamma} D^{\beta} u(s)\right) d s \\
& \pm D^{\beta} C(t) u^{0}+D^{\beta-1} C(t) u^{1}+\int_{0}^{t} D^{\beta-1} C(t-s) f\left(s, u(s), s^{\gamma} D^{\beta} u(s)\right) d s .
\end{aligned}
$$

Therefore, by our hypotheses on $u^{0}, u^{1}$ and $f$ we have $t^{\gamma} D^{\beta} \psi \in C[0, T]$. Put $u_{0}(t):=C(t) u^{0}+S(t) u^{1}$ and define for $n=1,2, \ldots$

$$
u_{n}(t):=C(t) u^{0}+S(t) u^{1}+\int_{0}^{t} S(t-s) f\left(s, u_{n-1}(s), s^{\gamma} D^{\beta} u_{n-1}(s)\right) d s .
$$


Clearly, $u_{n}, n=0,1,2, \ldots$ are well-defined, $u_{n} \in C_{\beta, \gamma}^{R L}([0, T])$ and

$$
\left\|u_{1}(t)-u_{0}(t)\right\| \leq K_{1}, \quad t \in[0, T]
$$

where $K_{1}=\tilde{N} T W$ and by Lemma 5 , we have

$$
\begin{aligned}
\left\|D^{\beta} u_{1}(t)-D^{\beta} u_{0}(t)\right\| & \leq \int_{0}^{t}\left|D^{\beta-1} C(t-s)\right|\left\|f\left(s, u_{0}(s),\left(s^{\gamma} D^{\beta} u_{0}\right)(s)\right)\right\| d s \\
& \leq K W T=: K_{2}, \quad t \in(0, T]
\end{aligned}
$$

where $K$ is a bound for $\left|D^{\beta-1} C(t)\right|$ and $W$ is a bound for $f$ (so $K_{1}$ and $K_{2}$ depend on $T$ ). Moreover, (3.4) and (3.5) imply

$$
\begin{aligned}
& \left\|u_{2}(t)-u_{1}(t)\right\| \\
& =\left\|\int_{0}^{t} S(t-s)\left[f\left(s, u_{1}(s), s^{\gamma} D^{\beta} u_{1}(s)\right)-f\left(s, u_{0}(s), s^{\gamma} D^{\beta} u_{0}(s)\right)\right] d s\right\| \\
& \quad \leq \tilde{N} \int_{0}^{t} F(s)\left[\left\|u_{1}(s)-u_{0}(s)\right\|+s^{\gamma}\left\|D^{\beta} u_{1}(s)-D^{\beta} u_{0}(s)\right\|\right] d s \\
& \quad \leq \tilde{N}\left(K_{1}+T^{\gamma} K_{2}\right) t \sup _{s \in[0, T]} F(s), \quad t \in[0, T]
\end{aligned}
$$

and

$$
\begin{aligned}
& \left\|D^{\beta} u_{2}(t)-D^{\beta} u_{1}(t)\right\| \\
& \quad \leq \int_{0}^{t}\left|D^{\beta-1} C(t-s)\right|\left\|f\left(s, u_{1}(s), s^{\gamma} D^{\beta} u_{1}(s)\right)-f\left(s, u_{0}(s), s^{\gamma} D^{\beta} u_{0}(s)\right)\right\| d s \\
& \quad \leq \int_{0}^{t}\left|D^{\beta-1} C(t-s)\right| F(s)\left[\left\|u_{1}(s)-u_{0}(s)\right\|+s^{\gamma}\left\|D^{\beta} u_{1}(s)-D^{\beta} u_{0}(s)\right\|\right] d s \\
& \quad \leq K\left(K_{1}+T^{\gamma} K_{2}\right) t \sup _{s \in[0, T]} F(s), \quad t \in(0, T] .
\end{aligned}
$$

The next step is

$$
\begin{aligned}
& \left\|u_{2}(t)-u_{1}(t)\right\| \\
& =\left\|\int_{0}^{t} S(t-s)\left[f\left(s, u_{2}(s), s^{\gamma} D^{\beta} u_{2}(s)\right)-f\left(s, u_{1}(s), s^{\gamma} D^{\beta} u_{1}(s)\right)\right] d s\right\| \\
& \leq \tilde{N} \int_{0}^{t} F(s)\left[\left\|u_{2}(s)-u_{1}(s)\right\|+s^{\gamma}\left\|D^{\beta} u_{2}(s)-D^{\beta} u_{1}(s)\right\|\right] d s \\
& \leq \tilde{N}\left(K_{1}+T^{\gamma} K_{2}\right)\left[\sup _{s \in[0, T]} F(s)\right]^{2}\left[\tilde{N}+K T^{\gamma}\right] \frac{t^{2}}{2} \\
& \leq 2 \tilde{N}\left(K_{1}+T^{\gamma} K_{2}\right)\left[\sup _{s \in[0, T]} F(s)\right]^{2} \max \left\{\tilde{N}, K T^{\gamma}\right\} \frac{t^{2}}{2} \\
& \leq\left(K_{1}+T^{\gamma} K_{2}\right)\left[\max \left\{\tilde{N}, K T^{\gamma}\right\} \sup _{s \in[0, T]} F(s)\right]^{2} 2 \frac{t^{2}}{2}, \quad t \in[0, T]
\end{aligned}
$$


and

$$
\begin{aligned}
& \left\|D^{\beta} u_{3}(t)-D^{\beta} u_{2}(t)\right\| \\
& \quad \leq \int_{0}^{t}\left|D^{\beta-1} C(t-s)\right|\left\|f\left(s, u_{2}(s), s^{\gamma} D^{\beta} u_{2}(s)\right)-f\left(s, u_{1}(s), s^{\gamma} D^{\beta} u_{1}(s)\right)\right\| d s \\
& \quad \leq \int_{0}^{t}\left|D^{\beta-1} C(t-s)\right| F(s)\left[\left\|u_{2}(s)-u_{1}(s)\right\|+s^{\gamma}\left\|D^{\beta} u_{2}(s)-D^{\beta} u_{1}(s)\right\|\right] d s \\
& \quad \leq K\left(K_{1}+T^{\gamma} K_{2}\right)\left[\sup _{s \in[0, T]} F(s)\right]^{2} \max \left\{\tilde{N}, K T^{\gamma}\right\} 2 \frac{t^{2}}{2}, \quad t \in(0, T] .
\end{aligned}
$$

Therefore

$$
\begin{aligned}
& t^{\gamma}\left\|D^{\beta} u_{3}(t)-D^{\beta} u_{2}(t)\right\| \\
& \quad \leq\left(K_{1}+T^{\gamma} K_{2}\right)\left[\max \left\{\tilde{N}, K T^{\gamma}\right\} \sup _{s \in[0, T]} F(s)\right]^{2} 2 \frac{t^{2}}{2}, \quad t \in(0, T] .
\end{aligned}
$$

Continuing in this way, we find

$$
\begin{aligned}
& \max \left\{\left\|u_{n+1}-u_{n}\right\|, t^{\gamma}\left\|D^{\beta} u_{n+1}-D^{\beta} u_{n}\right\|\right\} \\
& \quad \leq\left(\max \left\{\tilde{N}, T^{\gamma} K\right\} \sup _{s \in[0, T]} F(s)\right)^{n} \frac{\left(K_{1}+T^{\gamma} K_{2}\right) 2^{n-1} T^{n}}{n !}, \quad n=1,2, \ldots
\end{aligned}
$$

Therefore $\lim _{n \rightarrow \infty} u_{n}(t)=u(t)$ and $\lim _{n \rightarrow \infty} t^{\gamma} D^{\beta} u_{n}(t)=t^{\gamma} v(t)$ exist uniformly in $[0, T]$ and $t^{\gamma} D^{\beta} u(t)=t^{\gamma} v(t), t \in[0, T]$. The limit $u(t)$ satisfies $(2.2)$. This follows from

$$
\begin{aligned}
& \left\|u(t)-C(t) u^{0}-S(t) u^{1}-\int_{0}^{t} S(t-s) f\left(s, u(s), s^{\gamma} D^{\beta} u(s)\right) d s\right\| \\
& \leq\left\|u(t)-u_{n+1}(t)\right\|+\tilde{N} \int_{0}^{t}\left\|f\left(s, u_{n}(s), s^{\gamma} D^{\beta} u_{n}(s)\right)-f\left(s, u(s), s^{\gamma} D^{\beta} u(s)\right)\right\| d s \\
& \leq\left\|u(t)-u_{n+1}(t)\right\|+\tilde{N} \int_{0}^{t} F(s)\left[\left\|u_{n}(s)-u(s)\right\|+s^{\gamma}\left\|D^{\beta} u_{n}(s)-D^{\beta} u(s)\right\|\right] d s .
\end{aligned}
$$

To see the uniqueness, assume that there are two mild solutions $u_{1}(t)$ and $u_{2}(t)$, then

$$
\begin{aligned}
& \left\|u_{1}(t)-u_{2}(t)\right\| \leq \tilde{M}\left\|u_{1}^{0}-u_{2}^{0}\right\|+\tilde{N}\left\|u_{1}^{1}-u_{2}^{1}\right\| \\
& \quad+\tilde{N} \int_{0}^{t} F(s)\left[\left\|u_{1}(s)-u_{2}(s)\right\|+s^{\gamma}\left\|D^{\beta} u_{1}(s)-D^{\beta} u_{2}(s)\right\|\right] d s, \quad t \in[0, T]
\end{aligned}
$$

and

$$
\begin{aligned}
& \left\|D^{\beta} u_{1}(t)-D^{\beta} u_{2}(t)\right\| \leq\left|D^{\beta} C(t)\right|\left\|u_{1}^{0}-u_{2}^{0}\right\|+\left|D^{\beta-1} C(t)\right|\left\|u_{1}^{1}-u_{2}^{1}\right\| \\
& \quad+\int_{0}^{t} K F(s)\left[\left\|u_{1}(s)-u_{2}(s)\right\|+s^{\gamma}\left\|D^{\beta} u_{1}(s)-D^{\beta} u_{2}(s)\right\|\right] d s, \quad t \in(0, T] .
\end{aligned}
$$


Gronwall inequality implies that

$$
\begin{aligned}
& \left\|u_{1}(t)-u_{2}(t)\right\|+t^{\gamma}\left\|D^{\beta} u_{1}(t)-D^{\beta} u_{2}(t)\right\| \\
& \quad \leq C_{1}\left(\left\|u_{1}^{0}-u_{2}^{0}\right\|+\left\|u_{1}^{1}-u_{2}^{1}\right\|\right) \exp \left(C_{2} \int_{0}^{T} F(s) d s\right), \quad t \in[0, T]
\end{aligned}
$$

for some positive constants $C_{1}$ and $C_{2}$ (depending on $T$ ). Hence $u_{1}(t) \equiv u_{2}(t)$.

Remark 1. If we are to prove the existence of mild solutions only then we would not have bothered ourselves with the term $t^{\gamma}$. This means that our previous result holds for $\gamma=0$, that is in $C_{\beta, 0}^{R L}([0, T])$ and with initial data in $E_{\beta, 0} \times X$ (see (3.1) and (3.2)). The term $t^{\gamma}$ is there to avoid the singularity of the fractional derivative in case of smoothness of the solution and the initial data. In particular, this would be the case when we deal with classical solutions (see next section).

\section{Classical Solutions}

In this section we prove the existence and uniqueness of classical solutions to problem (1.1).

Theorem 2. Suppose that $(\mathrm{H} 1)-(\mathrm{H} 2)$ hold and $\gamma \geq \beta$. Assume further that $u^{0} \in D(A), u^{1} \in E$ and $f$ is an E-valued function. Then, the solution $u(t)$ of problem (2.2) is twice continuously differentiable and satisfies (1.1) on $[0, T]$ for some $T>0$.

Proof. Thanks to Lemma 2, $\left(u^{0}, u^{1}\right) \in D(A) \times E$ implies that $\left(u^{0}, u^{1}\right) \in$ $E_{\beta, \gamma} \times E_{\beta-1, \gamma}$ with $\gamma \geq \beta$ and, as $f$ is an $E$-valued function, $t^{\beta-1} D^{\beta-1} C(t) f$ is continuous and therefore

$$
t^{\gamma} \int_{0}^{t} D^{\beta-1} C(s) f(s) d s=t^{\gamma} \int_{0}^{t} \frac{s^{\beta-1}}{s^{\beta-1}} D^{\beta-1} C(s) f(s) d s \leq \tilde{K} \frac{t^{\gamma+2-\beta}}{2-\beta} .
$$

Hence, the previous theorem applies and we have existence and uniqueness of a mild solution i.e.

$$
u(t)=C(t) u^{0}+S(t) u^{1}+\int_{0}^{t} S(t-s) f\left(s, u(s), s^{\gamma} D^{\beta} u(s)\right) d s, \quad t \in[0, T] .
$$

By Lemma 2, we may write

$$
u^{\prime}(t):=A S(t) u^{0}+C(t) u^{1}+\int_{0}^{t} C(t-s) f\left(s, u(s), s^{\gamma} D^{\beta} u(s)\right) d s .
$$

Let us now consider the expression

$$
\begin{aligned}
\varphi(t)= & A C(t) u^{0}+A S(t) u^{1}+f\left(t, u(t),\left(s^{\gamma} D^{\beta} u\right)(t)\right) \\
& +\int_{0}^{t} A S(t-s) f\left(s, u(s), s^{\gamma} D^{\beta} u(s)\right) d s, \quad t \in[0, T] .
\end{aligned}
$$


Observe that by our assumptions $u^{0} \in D(A), u^{1} \in E$ and Lemma 2 (iv) and (ii) the terms $C(t) A u^{0}$ and $A S(t) u^{1}$ are well-defined. Moreover, as $f$ is an $E$-valued function, it is clear that $\varphi(t)$ is a continuous function on $[0, T]$ (Lemma 2 (iii)) and $w(t)=u^{\prime}(t)$, where $w(t)=u^{1}+\int_{0}^{t} \varphi(s) d s, t \in[0, T]$. Finally, as $w(t)$ is continuously differentiable, the function $u(t)$ is twice continuously differentiable, $u(.) \in D(A)$ (see Lemma 3) and clearly it satisfies problem (1.1).

Remark 2. The regularity of solutions for the linear inhomogeneous equation $u^{\prime \prime}(t)=A u(t)+f(t)$ corresponding to (1.1) is studied in [26]. It has been proved that if $u^{0} \in D(A)$ and $u^{1} \in E$ then the mild solution $u(.) \in D(A), u$ is twice continuously differentiable and is a classical solution (See Prop. 2.4) (see also Prop. 3.6 for the nonlinear case $f(t, u)$ ), provided that $f$ is continuously differentiable (see also [25, Corollary 3.5]). In case $f$ is continuous an alternative to this condition (of continuous differentiability) is " $q(t)=\int_{0}^{t} S(t-s) f(s) d s$ twice continuously differentiable" (see Prop. 3.4) which is in fact a necessary and sufficient condition. If this function $q(t)$ is twice continuously differentiable then it is in $D(A)$ and $A q(t)=q^{\prime \prime}(t)-f(t)$ (see Lemma 3). Another alternative is " $q(t) \in D(A)$ and $A q(t)$ is a continuous function" (see [25, Prop. 3.6]). We also obtain the regularity of the mild solution when $f(t) \in D(A)$ and the function $A f(t) \in D(A)$ is continuous (this is a corollary to Prop. 3.6).

Example 1. Some special cases of the present problem are some fractionally damped wave problems and viscoelastic problems [16], the (fractional) telegraph equation [10, 21], the Langevin problem [12], the Szabo's model, the Webster-Lokshin model, etc.

As an example we consider the Cauchy problem

$$
\left\{\begin{array}{l}
u_{t t}(t)=u_{x x}(t, \xi)+g\left(t, \xi, u(t, \xi), t^{\gamma} D^{\beta} u(t, \xi)\right), \quad t>0,0 \leq \xi \leq \pi \\
u(t, 0)=u(t, \pi)=0, \quad t>0, \\
u(0, \xi)=u^{0}(\xi), \quad u_{t}(0, \xi)=u^{1}(\xi), \quad 0 \leq \xi \leq \pi
\end{array}\right.
$$

in the space $X=L^{2}([0, \pi])$. This problem can be reformulated in the abstract setting (1.1). To this end we define the operator $A y=y^{\prime \prime}$ with domain

$$
D(A):=\left\{y \in H^{2}([0, \pi]): y(0)=y(\pi)=0\right\} .
$$

The operator $A$ has a discrete spectrum with $-n^{2}, n=1,2, \ldots$ as eigenvalues and $z_{n}(s)=\sqrt{2 / \pi} \sin (n s), n=1,2, \ldots$ as their corresponding normalized eigenvectors. So we may write

$$
A y=-\sum_{n=1}^{\infty} n^{2}\left(y, z_{n}\right) z_{n}, \quad y \in D(A) .
$$

Since $-A$ is positive and self-adjoint in $L^{2}([0, \pi])$, the operator $A$ is the infinitesimal generator of a strongly continuous cosine family $C(t), t \in \mathbf{R}$ which has the form

$$
C(t) y=\sum_{n=1}^{\infty} \cos (n t)\left(y, z_{n}\right) z_{n}, \quad y \in X .
$$


The associated sine family is found to be

$$
C(t) y=\sum_{n=1}^{\infty} \frac{\sin (n t)}{n}\left(y, z_{n}\right) z_{n}, \quad y \in X .
$$

We assume

(A) $g: \mathbf{R}_{+} \times[0, \pi] \times \mathbf{R} \times \mathbf{R} \rightarrow \mathbf{R}$ is continuous and satisfies the Lipschitz condition

$$
\left|g\left(t, \xi, x_{1}, y_{1}\right)-g\left(t, \xi, x_{2}, y_{2}\right)\right| \leq G(t, \xi)\left(\left|x_{1}-x_{2}\right|+\left|y_{1}-y_{2}\right|\right)
$$

for $t>0, x_{1}, y_{1}, x_{2}, y_{2} \in \mathbf{R}, \xi \in[0, \pi]$ and some continuous real-valued function $G(t, \xi)$. Next, defining

$$
f\left(t, u(t), t^{\gamma} D^{\beta} u(t)\right)(\xi):=g\left(t, \xi, u(t, \xi), t^{\gamma} D^{\beta} u(t, \xi)\right),
$$

the problem (4.1) can be written abstractly as

$$
\left\{\begin{array}{l}
u^{\prime \prime}(t)=A u(t)+f\left(t, u(t), t^{\gamma} D^{\beta} u(t)\right), \quad t>0 \\
u(0)=u^{0} \in X, \quad u^{\prime}(0)=u^{1} \in X
\end{array}\right.
$$

that is in the form of problem (1.1). Its mild solution is given by

$$
u(t)=C(t) u^{0}+S(t) u^{1}+\int_{0}^{t} S(t-s) f\left(s, u(s), s^{\gamma} D^{\beta} u(s)\right) d s
$$

and (H2) is satisfied. Assuming further that $\left(u^{0}, u^{1}\right) \in E_{\beta, \gamma} \times E_{\beta-1, \gamma}$ and $f$ is an $E_{\beta-1}$-valued continuous function, the first theorem ensures the existence of a unique mild solution in the space $C_{\beta, \gamma}^{R L}([0, T])$.

Under the assumptions $u^{0} \in D(A)$ and $u^{1} \in E, \gamma \geq \beta$ and $f$ is an $E$-valued continuous function the second theorem ensures that the mild solution found in the first theorem is actually twice continuously differentiable and is a classical solution of problem (1.1).

\section{Acknowledgment}

The author is very grateful for the financial support provided by King Fahd University of Petroleum and Minerals through project No. IN 100007.

\section{References}

[1] R.L. Bagley and P.J. Torvik. A different approach to the analysis of viscoelastically damped structures. AIAA Journal, 21:741-748, 1983. http://dx.doi.org/10.2514/3.8142.

[2] R.L. Bagley and P.J. Torvik. On the appearance of the fractional derivative in the behavior of real material. J. Appl. Mech., 51:294-298, 1983.

[3] R.L. Bagley and P.J. Torvik. A theoretical basis for the application of fractional calculus to viscoelasticity. J. Rheology, 27:201-210, 1983.

http://dx.doi.org/10.1122/1.549724. 
[4] M. Bahaj. Remarks on the existence results for second-order differential inclusions with nonlocal conditions. J. Dyn. Control Syst., 15(1):27-43, 2009. http://dx.doi.org/10.1007/s10883-008-9059-3.

[5] L. Beghin and E. Orsingher. The telegraph process stopped at stable-distributed times and its connection with the fractional telegraph equation. Fract. Calc. Appl. Anal., 6(2):187-204, 2003.

[6] M. Benchohra and S.K. Ntouyas. Existence of mild solutions of second order initial value problems for delay integrodifferential inclusions with nonlocal conditions. Math. Bohem., 4(127):613-622, 2002.

[7] M. Benchohra and S.K. Ntouyas. Existence results for the semi-infinite interval for first and second order integrodifferential equations in Banach spaces with nonlocal conditions. Acta Univ. Palack. Olomuc. Fac. Rerum Natur. Math., 41:13-19, 2002.

[8] M. Benchohra and S.K. Ntouyas. Existence results for multivalued semilinear functional differential equations. Extracta Math., 18(1):1-12, 2003.

[9] L. Byszewski and V. Lakshmikantham. Theorems about the existence and uniqueness of a solution of a nonlocal abstract Cauchy problem in a Banach space. Appl. Anal., 40(1):11-19, 1991. http://dx.doi.org/10.1080/00036819008839989.

[10] R.C. Cascaval, E.C. Eckstein, C.L. Frota and J. Godstein. Fractional telegraph equations. J. Math. Anal. Appl., 276:145-159, 2002. http://dx.doi.org/10.1016/S0022-247X(02)00394-3.

[11] R. Gorenflo and S. Vessella. Abel integral equations: Analysis and applications. In A. Dold, B. Eckmann and F. Takens(Eds.), Lecture Notes in Math., Berlin, Heidelerg, New York, 1991. Springer-Verlag.

[12] K.S. Hedrich and A. Filipovski. Longitudinal creep vibrations of a fractional derivative order rheological rod with variable cross section. Facta Univ. Ser. Mech. Automat. Control Robot., 3:327-349, 2002.

[13] E.M. Hernandez, H.R. Henríquez and M.A. McKibben. Existence of solutions for second order partial neutral functional differential equations. Integral Equations Operator Theory, 62:191-217, 2008. http://dx.doi.org/10.1007/s00020-008-1618-1.

[14] M.E. Hernandez. Existence of solutions to a second order partial differential equation with nonlocal conditions. Electron. J. Differential Equations, 51(2003):1-10, 2003.

[15] A.A. Kilbas, H.M. Srivastava and J.J. Trujillo. Theory and applications of fractional differential equations. In Jan van Mill(Ed.), North-Holland Math. Stud., volume 204, Amsterdam, The Netherlands, 2006. Elsevier.

[16] M. Kirane and N.-e. Tatar. Exponential growth for fractionally damped wave equation. Z. Anal. Anwend., 22(1):167-178, 2003. http://dx.doi.org/10.4171/ZAA/1137.

[17] A.A. Lokshin. Wave equation with singular delayed time. Dokl. Akad. Nauk SSSR, 240:43-46, 1978. (in Russian)

[18] A.A. Lokshin and V.E. Rok. Fundamental solutions of the wave equation with delayed time. Dokl. Akad. Nauk SSSR, 239:1305-1308, 1978. (in Russian)

[19] K.S. Miller and B. Ross. An Introduction to the Fractional Calculus and Fractional Differential Equations. John Wiley and Sons, New York, 1993. 
[20] K.B. Oldham and J. Spanier. The Fractional Calculus. Academic Press, New York, London, 1974.

[21] E. Orsingher and L. Beghin. Time-fractional telegraph equations and telegraph processes with brownian time. Probab. Theory Related Fields, 128:141-160, 2004. http://dx.doi.org/10.1007/s00440-003-0309-8.

[22] I. Podlubny. Fractional Differential Equations, volume 198 of Math. Sci. Eng. Academic Press, San-Diego, 1999.

[23] S.G. Samko, A.A. Kilbas and O.I. Marichev. Fractional Integrals and Derivatives. Theory and Applications. Gordon and Breach, Yverdon, 1993.

[24] N.-e. Tatar. The existence of mild and classical solutions for a second-order abstract fractional problem. Nonlinear Anal., 73:3130-3139, 2010. http://dx.doi.org/10.1016/j.na.2010.06.085.

[25] C.C. Travis and G.F. Webb. Compactness, regularity and uniform continuity properties of strongly continuous cosine families. Houston J. Math., 3(4):555567, 1977.

[26] C.C. Travis and G.F. Webb. Cosine families and abstract nonlinear second order differential equations. Acta Math. Acad. Sci. Hungaricae, 32:76-96, 1978. http://dx.doi.org/10.1007/BF01902205.

[27] C.C. Travis and G.F. Webb. An abstract second order semilinear Volterra integrodifferential equation. SIAM J. Math. Anal., 10(2):412-424, 1979. http://dx.doi.org/10.1137/0510038. 